\title{
Experimental Study on Downhole Oil-water Separation Hydrocyclone
}

\author{
$\mathrm{Xu}$ Yan, Yuan Lin, Wang Zunce*, Li Sen, Zhang Jinglong and Ke Lin \\ Mechanical Science and Engineering College, Northeast Petroleum University, Daqing 163318, P.R. China
}

\begin{abstract}
In the process of downhole oil-water separation, the traditional liquid-liquid separation hydrocyclone (LLSH) is used in conjunction with screw pumps, which makes the hydrocyclone rotating around its own axis. The rotation of wall of hydrocyclone affects its internal flow characteristics and separation properties directly. The orthogonal experiment of the downhole oil-water separation hydrocyclone (DOWSH) is designed to analyze the effect of flowrate, rotating speed and split ratio on separation efficiency and pressure drop, The primary and secondary factors of operation parameters have been studied, and the optimum condition and reasonable working range of DOWSH have been obtained. It provides reliable basis for process of practical application of DOWSH so as to guide the production.
\end{abstract}

Keywords: Downhole oil-water separation hydrocyclone, orthogonal experiment, rotating speed.

\section{INTRODUCTION}

The technology of downhole oil-water hydrocyclone separation has a very positive significance in reducing the cost of the produced fluid lift, the ground water treatment, and the moisture content of produced fluid [1]. It also has inestimable value for the extension of the exploitation of the old oil wells [2]. In the process of downhole oil-water separation, the traditional liquid-liquid separation hydrocyclone (LLSH) is used in conjunction with screw pumps, which makes the hydrocyclone rotating around its own axis.

Since Martin Thew et al. [3] designed the first LLSH, international and domestic scholars have conducted studies on the separation mechanism and the application of LLSH. Wang et al. $[4,5]$ carried out a lot of experimental research of LLSH, and studied the effect of structure parameters, pressure loss and operating parameters on its separation performance. The effect of velocity fluctuations on the separation efficiency of LLSH have been investigated using numerical simulations and experiments [6]. The effect of the inlet dimensions of LLSH on the internal flow pattern have been studied $[7,8]$.

The flow in the downhole oil-water separation hydrocyclone (DOWSH) is rotating turbulence coupled with spiral flow and rotating wall, the internal flow pattern, separation efficiency and pressure characteristics are influenced by many factors, and the research work is complicated. So the study on the flow rule of DOWSH is necessary. However, for the impact of rotating wall on separation performance, there is still no specific theory in engineering.

In this paper, the experimental apparatus of DOWSH is designed, the experiment are conducted to investigate its structure and separation properties. The optimum condition and reasonable working range of DOWSH can be studied according to separate efficiently. It provides reliable basis for application of DOWSH.

\section{EXPERIMENTAL DEVICE}

The main structure of DOWSH is the same with LLSH, as shown in Fig. (1). Combined with downhole conditions, the indoor experimental device of DOWSH is designed, it is mainly composed of the transmission, the separation and the auxiliary. The transmission part is composed of the transmission shaft, the hollow driving shaft and the synchronous rotating cylinder etc, which transfer the power of the motor to hydrocyclone. The separation part is the hydrocyclone to realize the separation of oil-water two phase. The auxiliary part includes the motor, the coupling and the stationary outer cylinder etc., the structure is shown in Fig. (2).

In the process of the experiment, the hydrocyclone rotates around its own axis by the motor driven, the oil-water mixture flows into the annular space between the hydrocyclone and the outer cylinder from the inlet, through both two sides tangential inlets flows into the hydrocyclone, the oilwater mixture flow in the hydrocyclone with rotating wall, and is separated, the separated oil outflows through the overflow, the separated water outflows through the underflow.

\section{EXPERIMENTAL PROCESS}

To study the influence of operating parameters (flowrate, rotating speed, split ratio) of DOWSH on the separation efficiency and pressure drop, The experiment process has been designed as shown in Fig. (3), the process are consist of the fluid supply unit, metering unit, oil and water mixing device, experimental device of DOWSH, liquid recovery unit. Considering that the centrifugal pump would have an impact of sheared emulsification on and make droplets. The screw pump is used in order to prevent droplets dispersing and recombining and achieve good mixed effect. Then the oil and water mixture flow into the hydrocyclone of rotating wall, inlet pressure, outlet pressure and flowrate are metered respectively. The picture of flow chart of experiment is as shown in Fig. (4). 


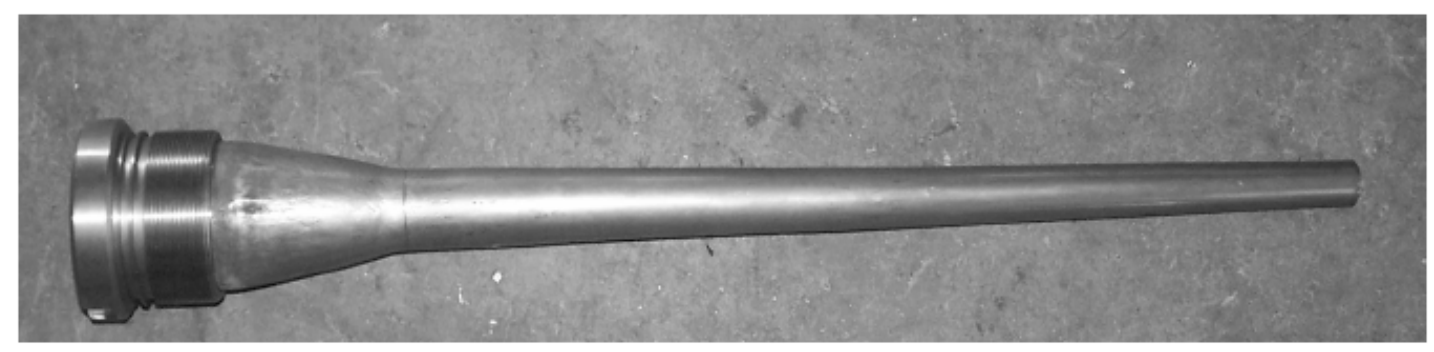

Fig. (1). The picture of hydrocyclone.

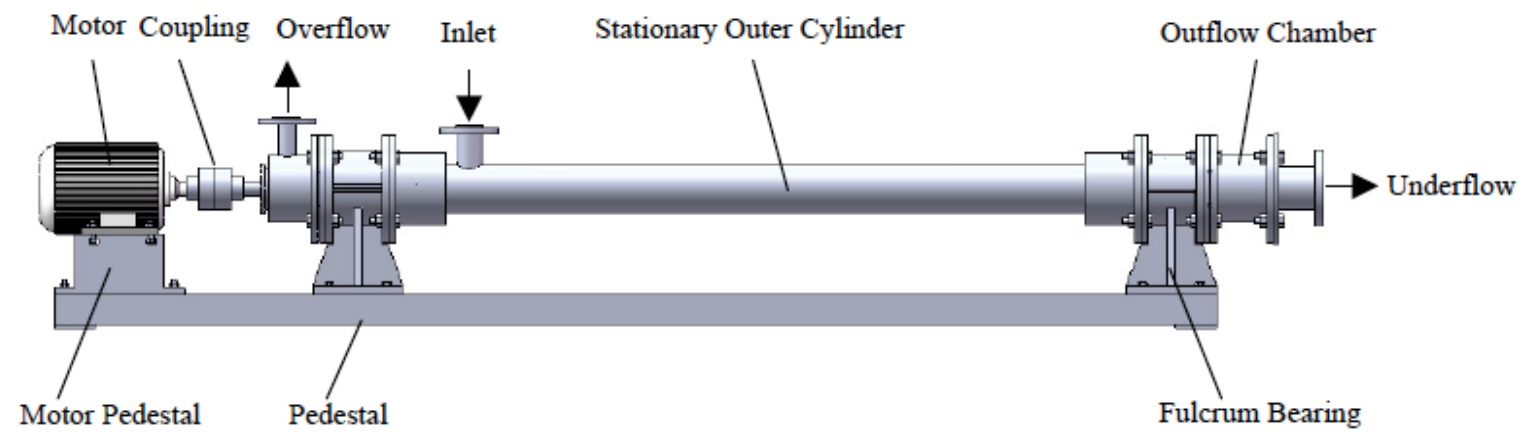

Fig. (2). Sketch of the experimental device of DOWSH.



Fig. (3). Flow chart of experiment of DOWSH.

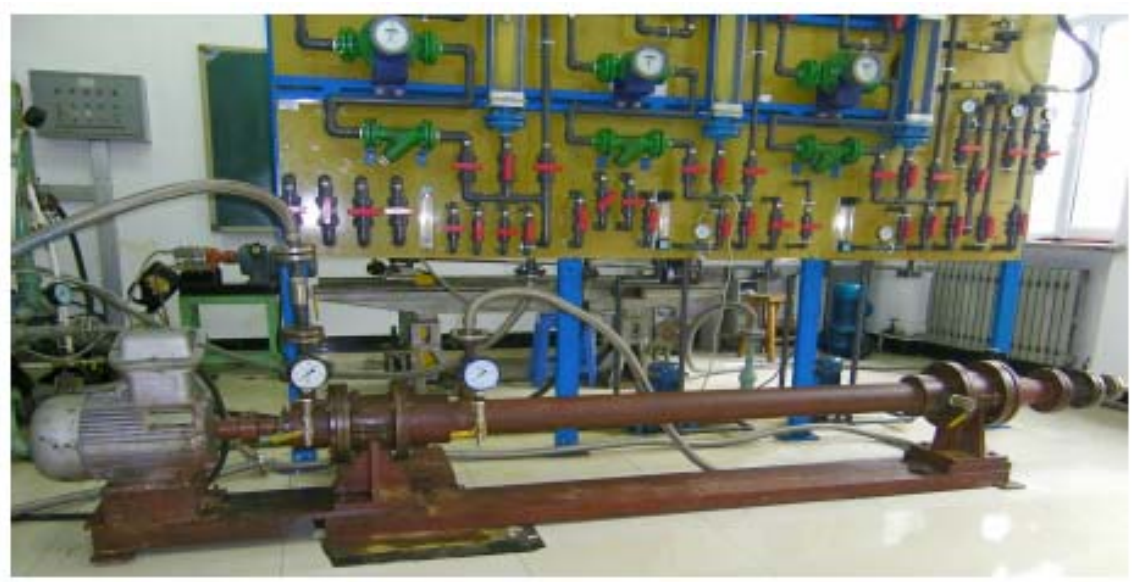

Fig. (4). The picture of flow chart of experiment of DOWSH. 
4. ANALYSIS OF PRIMARY AND SECONDARY FACTORS OF INFLUENCE OF OPERATING PARAMETERS ON SEPARATION CHARACTERISTICS

\subsection{Parameters of Experiment}

(1) Split Ratio

$F=Q_{u} / Q_{i}$

The liquid in DOWSH discharges from overflow and underflow, and split ratio is used to describe the proportional relationship between the overflow and the underflow of the flowrate. We can control the split ratio through adjusting the underflow flowrate in the experiment.

\section{(2) Separation Efficiency}

$$
E_{j}=1-\frac{C_{d}}{C_{i}}
$$

The equation (2) describes the actual treating results of DOWSH, that is to say, when no separation effect is achieved, the separation efficiency is 0 , while the complete separation is accomplished, the separation efficiency is 1 . The separation efficiency is given by sampling at the inlet and the underflow, extracting, and measuring the volume fraction of water.

$\mathrm{C}_{\mathrm{i}}, \mathrm{C}_{\mathrm{d}} \longrightarrow$ Oil concentration of inlet and underflow of hydrocyclone, $(\%)$.

(3) Pressure Drop

$\Delta p_{d}=p_{i}-p_{d}$

In this paper the split ratio is determined as 5\%-15\% and the flowrate of underflow is large, so the underflow pressure drop $\Delta p_{d}$ represents the major pressure loss. In the separation process of DOWSH, the fuild depends on the pressure loss to acquire energy, so, if we want to obtain the same separation efficiency at the same flowrate, the more lower the pressure drop is, the more less the energy loss is.

\subsection{The Design of the Experiment}

The main diameter of experimental DOWSH is $28 \mathrm{~mm}$, the large cone angle is $20^{\circ}$, the small cone angle is $1.5^{\circ}$.

Using oil as the dispersed phase, the density is about 860 $\mathrm{kg} / \mathrm{m}^{3}$. Using water as the continuous phase, density is $1000 \mathrm{~kg} / \mathrm{m}^{3}$

Table 1. Factors and levels for experiment.

\begin{tabular}{|c|c|c|c|}
\hline Level & Flowrate $\left(\mathbf{m}^{\mathbf{3}} / \mathbf{h}\right)$ & Rotating Speed (r/min) & Split Ratio (\%) \\
\hline \hline 1 & 2.0 & 0 & 5 \\
\hline 2 & 2.5 & 100 & 10 \\
\hline 3 & 3.0 & 200 & 12 \\
\hline 4 & 3.5 & 300 & 15 \\
\hline
\end{tabular}

Table 2. Results of the orthogonal experiment.

\begin{tabular}{|c|c|c|c|c|c|c|c|c|c|c|c|}
\hline No. & $\mathbf{A}$ & $\mathbf{B}$ & $\mathbf{C}$ & $\begin{array}{c}\mathbf{E}_{\mathbf{j}} \\
(\boldsymbol{\%})\end{array}$ & $\begin{array}{c}\text { Pressure Drops } \\
(\mathbf{K p a})\end{array}$ & No. & $\mathbf{A}$ & $\mathbf{B}$ & $\mathbf{C}$ & $\begin{array}{c}\mathbf{E}_{\mathbf{j}} \\
(\boldsymbol{\%})\end{array}$ & $\begin{array}{c}\text { Pressure Drops } \\
(\text { Kpa) }\end{array}$ \\
\hline \hline 1 & 1 & 1 & 1 & 70.57 & 82 & 14 & 3 & 4 & 1 & 84.88 & 210 \\
\hline 2 & 1 & 2 & 2 & 73.7 & 95 & 15 & 3 & 5 & 2 & 86.00 & 201 \\
\hline 3 & 1 & 3 & 3 & 79.24 & 106 & 16 & 4 & 1 & 4 & 93.40 & 216 \\
\hline 4 & 1 & 4 & 4 & 82.46 & 124 & 17 & 4 & 2 & 5 & 94.82 & 215 \\
\hline 5 & 1 & 5 & 5 & 85.25 & 126 & 18 & 4 & 3 & 1 & 88.24 & 238 \\
\hline 6 & 2 & 1 & 2 & 78.60 & 119 & 19 & 4 & 4 & 2 & 90.56 & 243 \\
\hline 7 & 2 & 2 & 3 & 80.34 & 126 & 21 & 4 & 5 & 3 & 91.17 & 25 \\
\hline 8 & 2 & 3 & 4 & 82.68 & 138 & 22 & 5 & 2 & 1 & 78.32 & 262 \\
\hline 9 & 2 & 4 & 5 & 84.14 & 151 & 23 & 5 & 3 & 2 & 80.12 & 286 \\
\hline 10 & 2 & 5 & 1 & 80.38 & 174 & 24 & 5 & 4 & 3 & 85.24 & 318 \\
\hline 11 & 3 & 1 & 3 & 85.25 & 165 & 25 & 5 & 5 & 4 & 87.36 & 330 \\
\hline 12 & 3 & 2 & 4 & 88.20 & 168 & 195 & & & & 335 \\
\hline 13 & 3 & 3 & 5 & 92.57 & & & & \\
\hline
\end{tabular}


Table 3. Sum of factors in different levels.

\begin{tabular}{|c|c|c|c|c|c|c|}
\hline \multirow{2}{*}{ Level } & \multicolumn{3}{|c|}{ Separation Efficiency } & \multicolumn{3}{c|}{ Pressure Drops } \\
\cline { 2 - 7 } & A & B & C & A & B & 844 \\
\hline \hline K1 & 391.22 & 416.36 & 402.39 & 533 & 790 \\
\hline K2 & 406.14 & 415.38 & 415.38 & 708 & 990 \\
\hline K3 & 436.90 & 422.85 & 421.24 & 939 & 1167 & 985 \\
\hline K4 & 458.19 & 427.28 & 434.10 & 1531 & 1091 & 981 \\
\hline K5 & 419.58 & 430.16 & 445.32 & 949 \\
\hline
\end{tabular}

Table 4. Analysis of variance.

\begin{tabular}{|c|c|c|c|c|c|c|c|c|c|c|}
\hline \multirow{2}{*}{$\begin{array}{l}\text { Soruce of } \\
\text { Variation }\end{array}$} & \multicolumn{2}{|c|}{$\begin{array}{c}\text { Sum of Squares of } \\
\text { Deviations }\end{array}$} & \multirow{2}{*}{$\begin{array}{c}\text { Degrees of } \\
\text { freedom } \\
f\end{array}$} & \multicolumn{2}{|c|}{ Quadratic Mean $S / f$} & \multicolumn{2}{|c|}{$\mathbf{F}$} & \multirow{2}{*}{ Critical Value } & \multicolumn{2}{|c|}{ Significance } \\
\hline & $\mathbf{S}_{\mathbf{e}}$ & $\mathbf{S}_{\mathbf{P}}$ & & e & $\mathbf{P}$ & e & $\mathbf{P}$ & & e & $\mathbf{P}$ \\
\hline A & 547.14 & 122789.4 & 4 & 136.79 & 30697.35 & 53.43 & 433.15 & $F_{\alpha=0.01}(4,12)=5.41$ & $(* *)$ & $(* *)$ \\
\hline B & 34.00 & 9025.84 & 4 & 8.5 & 2256.46 & 3.32 & 31.84 & & & $(* *)$ \\
\hline E (Error) & 30.76 & 850.28 & 12 & 2.56 & 70.87 & & & & & \\
\hline $\mathrm{T}$ (Total) & 860.71 & 123502 & 24 & & & & & & & \\
\hline
\end{tabular}

Analysis of variance is conduced for the experimental data, the results show in Table 4 . The $(* *)$ signify the parameter is significant under 0.01 significant level.

Flowrate, rotating speed and split ratio are three major factors influenced on separation characteristics, the orthogonal experiment is designed to analyze these three factors. Flowrate (A factor), rotating speed (B factor), split ratio (C factors) are arranged, five levels are designed, and specific data is shown in Table $\mathbf{1}$.

\subsection{Analysis of Experimental Results}

Without considering the interaction between various factors, according to the orthogonal experiment method 25 sets of experiment has been arranged. Experimental designs and results of orthogonal experimental are shown in Table 2 and Table 3. Where Ej $(j=1,2 \ldots, 25)$ is the simplified separation efficiency, $Q$ is the sum of the squares of $\mathrm{Ej}, \mathrm{Ki}(\mathrm{i}=1,2 \ldots 5)$ is the sum of $\mathrm{Ej}$ of the levels number $\mathrm{i}, \mathrm{T}$ is the sum of squared difference.

The Table 4 shows that $\mathrm{A}$ and $\mathrm{C}$ factors affect separation efficiency much significantly, A and B factors effect on pressure drop much significantly through analysis of $F$ value and critical value. The order of effecting on the separation efficiency is flowrate $>$ split ratio > rotating speed, the optimal solution is for $\mathrm{A}_{4} \mathrm{~B}_{5} \mathrm{C}_{5}$. The order of affecting of pressure drop is flowrate $>$ rotating speed $>$ split ratio, the optimal solution is for $\mathrm{A}_{1} \mathrm{~B}_{1} \mathrm{C}_{5}$. The two indicators of the optimal implementation scheme is different, but we can already see from the test data, within the scope of the study the pressure drop is less than $300 \mathrm{kPa}$, so that the pressure loss have less impact on system operation. Therefore, the optimal scheme of operating parameters can be selected according to the separation efficiency target.
Since there is no $A_{4} B_{5} C_{5}$ in this scheme, and $A_{4} B_{2} C_{5}$ is the highest separation efficiency scheme in orthogonal test, in order to verify the inspection of the two schemes, 10 groups of experiments were carried out respectively. The results showed that the separation efficiency reaches $90 \%$ or more, B factor has little influence on the separation efficiency obtained in test analysis. So the optimal scheme is that the flowrate is $3.5 \mathrm{~m}^{3} / \mathrm{h}$, the split ratio is $15 \%$, and the rotating speed can be arbitrarily selected within the scope of this study.

\section{CONCLUSION}

1) The order of effecting on the separation efficiency is flowrate $>$ split ratio > rotating speed, the optimal solution is for $\mathrm{A}_{4} \mathrm{~B}_{5} \mathrm{C}_{5}$. In which flowrate and split ratio compared with the rotating speed have significantly impact on the separation efficiency.

2) The order of affecting of pressure drop is flowrate > rotating speed > split ratio, the optimal solution is for $\mathrm{A}_{1} \mathrm{~B}_{1} \mathrm{C}_{5}$. within the scope of the study the pressure drop is less than $300 \mathrm{kPa}$, so that the pressure loss have less impact on system operation. Therefore, the optimal scheme of operating parameters can be selected according to the separation efficiency target.

3) The optimal scheme is that the flowrate is $3.5 \mathrm{~m}^{3} / \mathrm{h}$, the split ratio is $15 \%$, and the rotating speed can be arbitrarily selected within the scope of this study. 


\section{CONFLICT OF INTEREST}

The authors confirm that this article content has no conflict of interest.

\section{ACKNOWLEDGEMENTS}

This research was supported by the National Natural Science Foundation of China (11172061 and 11402051), National Torch Program (2013GH530190) and the Postdoctoral Fund of the Heilongjiang Province of China (LBH-Z12273).

\section{REFERENCES}

[1] J. Yue, and C. Qi, "New development of downhole oil-water separation technology", Oil and Gas Field Ground Engineering, vol. 22, pp. 56-57, 2003.

[2] Y. Li, Numerical Simulation and Optimal Design of Downhole Oilwater Hydrocyclone Separator. China University Of Petroleum, China, 2008.
[3] D.A. Colman, and M.T. Thew, "Correlation of separation results from light dispersion hydrocyclone", Chemical Engineering Research and Design, vol. 61, pp. 233-240, 1983.

[4] Z. Wang, L. Zhao, F. Li, and M. Jiang, "Study on flow field and separation characteristic of Liquid-liquid hydrocyclone (6) - the influence of structure parameters on separation characteristics", Chemical Equipment Technology, vol. 21, pp. 14-16, 2000

[5] Z. Wang, Y. Gao, F. Lv, X. Liu, and F. Li, "The influence of entrance structure parameters on the pressure characteristic of liquidliquid hydrocyclone", Fluid Machinery, vol. 31, pp. 16-18, 2003.

[6] S. K. Shukla, P. Shukla, and P. Ghosh, "The effect of modeling of velocity fluctuations on prediction of collection efficiency of cyclone separators", Applied Mathematical Modelling, vol. 37, pp. 5774-5789, 2013.

[7] K. Elsayed, and C. Lacor, "The effect of cyclone inlet dimensions on the flow pattern and performance", Applied Mathematical Modelling, vol. 35, pp. 1952-1968, 2011.

[8] F. Qian, and Y. Wu, "Effects of inlet section angle on separation performance of a cyclone", Chemical Engineering Research and Design, vol. 87, pp. 1567-1572, 2009.

(c) Yan et al.; Licensee Bentham Open.

This is an open access article licensed under the terms of the Creative Commons Attribution Non-Commercial License (http://creativecommons.org/licenses/by-nc/3.0/) which permits unrestricted, non-commercial use, distribution and reproduction in any medium, provided the work is properly cited. 